G. M. C.; Silva, C. P.; Leite, A. P.; Oliveira Neto, M. B. Zoneamento agroecológico do Nordeste: diagnóstico e prognóstico. CD-ROM. Recife/ Petrolina: Embrapa Solos e Embrapa Semi-Árido. 2000.

10. Leal, I. R.; Lopes, A. V.; Machado, I. C.; Tabarelli, M. "Plant-animal interactions in the Caatinga: overview and perspectives" In: Silva, J. M. C.; Leal, I. R.; Tabarelli, M. (eds.) Caatinga. The largest tropical dry forest region in South America. Cham: Springer International Publishing. 2017, p. 255-278.

11. Gariglio, M. A.; Sampaio, E. V. S. B.; Cestaro, L. A.; Kageyama, P. Y. Uso sustentável e conservação dos recursos florestais da Caatinga. Brasília: Serviço Florestal Brasileiro. 2010.

12. Silva J. M. C.; Barbosa, L. C. B.; Pinto, L. P. S.; Chennault, C. M. "Sustainable development in the Caatinga" In: Silva, J. M. C.; Leal, I. R.; Tabarelli, M. (eds.) Caatinga. The largest tropical dry forest region in South America. Cham: Springer International Publishing. 2017c, p. 445-460.

13. Sampaio, E. V. S. B. "Overview of the brazilian Caatinga". In: Bullock, S. H.; Mooney, H. A.; Medina, E. (eds.) Seasonally dry forests. Cambridge: Cambridge University Press. 1995, p. 35-58.

14. Guimarães Filho, C.; Góes, J. G. "Desempenho reprodutivo de caprinos na Caatinga sob diferentes taxas de lotação". Pesquisa Agropecuária Brasileira 1998, 23:309-314.

15. Leal, I. R.; Vicente, A.; Tabarelli, M. "Herbivoria por caprinos na Caatinga da região de Xingó: uma análise preliminar". In: Leal, I. R.; Tabarelli, M.; Silva, J. M. C. (eds.) Ecologia e conservação da Caatinga. Recife: Editora Universitária da UFPE. 2003, p. 695-715.

16. Melo, F.P. L. "The socio-ecology of the Caatinga: understanding how natural resource use shapes an ecosystem". In: Silva, J. M. C.; Leal, I. R.; Tabarelli, M. (eds.) Caatinga. The largest tropical dry forest region in South America. Cham: Springer International Publishing. 2017, p. 369-382.

17. Silva, J. M. C.; Barbosa, L. C. F. "Impact of human activities on the Caatinga". In: Silva, J. M. C.; Leal, I. R.; Tabarelli, M. (eds.) Caatinga. The largest tropical dry forest rin South America. Cham: Springer International Publishing. 2017d, p. 359-368.

18. Fonseca, C.R.; Antongiovanni, M.; Matsumoto, M.; Bernard, E.; Venticinque, E. M. "Conservation opportunities in the Caatinga". In:Silva, J. M. C.; Leal, I. R.; Tabarelli, M. (eds.) Caatinga. The largest tropical dry forest region in South America. Cham: Springer International Publishing. 2017, p. 429-444.

19. Torres, R. R.; Lapola, D. M.; Gamarra, N. R. L. "Future climate change in the Caatinga". In: Silva, J. M. C.; Leal, I. R.; Tabarelli, M. (eds.) Caatinga. The largest Trtpical dry forest region in South America. Cham: Springer International Publishing. 2017, p. 383-412.

20. Kasecker, T. P.; Ramos-Neto, M. B.; Silva, J. M. C.; Scarano, F. R. "Ecosystem-based adaptation to climate change: defining hotspot municipalities for policy design and implementation in Brazil". Mitigation and Adaptation Strategies to Global Change 2017. https://doi.org/10.1007/s11027-017-9768-6.

\section{OS ANIMAIS VERTEBRADOS DO BIOMA GAATINAA}

Adrian Antonio Garda, Marília Bruzzi Lion, Sérgio Maia de Queiroz Lima, Daniel Oliveira Mesquita, Helder Farias Pereira de Araujo e Marcelo Felgueiras Napoli

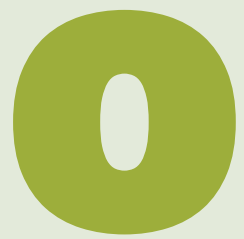

Brasil é um país megadiverso, mundialmente conhecido por suas exuberantes florestas tropicais. Devido à impressionante diversidade biológica nessas regiōes, áreas mais secas ou menos florestais, como a Caatinga, receberam menos atenção ao longo da história. De cientistas a leigos, de ambientalistas a tomadores de decisão, muitos conferiram maior importância, conscientemente ou não, às grandes florestas brasileiras. Com os zoólogos não foi diferente, porém mais especificamente na ornitologia (estudo das aves) e na mastozoologia (estudo dos mamíferos), áreas com grande contingente de pesquisadores que lidam com espécies mais carismáticas, foram produzidos os primeiros trabalhos sobre a fauna de vertebrados da região. Coincidentemente, esses grupos taxonômicos possuem as menores taxas de endemismo (espécies exclusivas) na Caatinga e riqueza de espécies moderadas quando comparado às florestas Amazônica e Atlântica. Ainda assim, mesmo entre esses grupos, o número total de espécies que habitam a Caatinga está longe de ser desprezível, como veremos. Tudo isso, aliado às paisagens semiáridas e aos piores índices de desenvolvimento humano levaram a Caatinga à marginalização intelectual e política, com resultados práticos temerosos: apenas $7,5 \%$ do bioma atualmente está sob alguma forma de proteção em unidades de conservação (mais de $98 \%$ na forma mais branda, de área de proteção ambiente - APA), sendo apenas 1,13\% em área de proteção integral [1]. Assim, durante muito tempo prevaleceu a visão de que a Caatinga teria baixa diversidade e pouca importância para a conservação da biodiversidade.

Nada mais longe da realidade: a Caatinga, assim como o resto do Brasil, é também megadiversa. A riqueza e o endemismo de vertebrados da Caatinga impressionam, ficando sem sombra de dúvida entre as regiōes semiáridas mais biodiversas do mundo. Além disso, é um bioma crucial para o entendimento do efeito das mudanças climáticas que ocorreram no passado sobre nosso continente e, portanto, chave para compreendermos os possíveis desdobramentos futuros das mudanças climáticas atuais. Ao longo dos 1,8 milhão de anos que correspondem ao Pleistoceno (entre 1,8 milhões e 11,7 mil anos atrás), os ciclos de glaciação e aquecimento fizeram com que as florestas tropicais se expandissem e se contraíssem. Os repetidos contatos entre Amazônia e Floresta Atlântica influenciaram esses dois biomas e contribuíram para a diversificação da fauna da Caatinga [2-6]. Hoje, como um grande museu, a história desses ciclos e da diversificação da Caatinga e de 
biomas vizinhos ainda pode ser vista nos enclaves de floresta úmida no centro e nas margens do bioma. $\mathrm{O}$ trabalho de gerações pioneiras e de novos pesquisadores vem, nas últimas décadas, revelando a riqueza, a história e a identidade da Caatinga.

Assim como diversas fisionomias caracterizam o Cerrado, a Floresta Atlântica e a Amazônia, a Caatinga é marcada por ambientes extremamente diversos. Essa variabilidade influenciou na biodiversidade local, permitindo o uso de sua área por espécies de biomas vizinhos e influenciando na evolução de linhagens exclusivas daquele bioma. A confusão entre o que chamamos de vegetação típica da Caatinga (florestas sazonalmente secas) e o bioma como um todo levou pesquisadores a excluir espécies de áreas úmidas das primeiras compilações acerca de sua riqueza e do seu endemismo. Um exemplo crítico do efeito dessa abordagem equivocada foi o número significativamente subestimado de espécies de anfíbios. A inclusão de áreas úmidas contribuiu para um aumento de $75 \%$ na riqueza conhecida de espécies de anfíbios da Caatinga (Tabela 1) e o aumento no esforço amostral com novos levantamentos de fauna elevou substancialmente a riqueza conhecida de serpentes [7], lagartos [6], peixes [5] e mesmo mamíferos [4].

Hoje, sabemos que algo próximo a 1.400 espécies de vertebrados ocorrem na Caatinga e 23\% destas são endêmicas (Figura 1), ou seja, somente ocorrem nesse bioma (Tabela 1). Aproximadamente metade das espécies de peixes e lagartos são endêmicas da Caatinga e a taxa de descrição de novas espécies de peixes, anfíbios e répteis ainda é muito alta. A título de exemplo, das 20 espécies de anfíbios endêmicas do bioma, 16 foram descritas depois de 2006! Igualmente, muitos novos registros e descrições de novas espécies, resultantes de intensos trabalhos de campo e de cuidadosos estudos taxonômicos, contribuíram para o aumento da diversidade conhecida de aves e mamíferos. O nosso conhecimento sobre a fauna de vertebrados da Caatinga aumentou muito nos últimos 20 anos, mas muito ainda há por ser feito. Aqui, oferecemos um panorama geral do conhecimento da riqueza, diversidade, ecologia e conservação dos animais vertebrados da Caatinga.

PEIXES Os peixes da Caatinga apresentados aqui correspondem somente às espécies registradas nos corpos d'água dentro do bioma, incluindo os enclaves úmidos e excluindo-se porções das bacias inseridas em outros biomas adjacentes. Essa ictiofauna (conjunto das espécies de peixes existente numa determinada região biogeográfica) é composta por uma mistura de espécies bastante diversa, de raias de água doce a peixes elétricos, abrigando desde espécies de grande porte que vivem em grandes rios perenes e que são usados na pesca comercial até peixes de menor porte e distribuição geográfica mais restrita, como bagres de caverna. Apesar da Caatinga possuir uma modesta rede hidrográfica de cursos d'água, boa parte intermitente, mais da metade das 371 espécies nativas da região são endêmicas [5]. Além disso, outras 32 espécies de peixes aguardam descrição formal pela ciência. No total, 386 espécies são conhecidas para o bioma (incluindo as exóticas introduzidas pela aquicultura, aquarismo
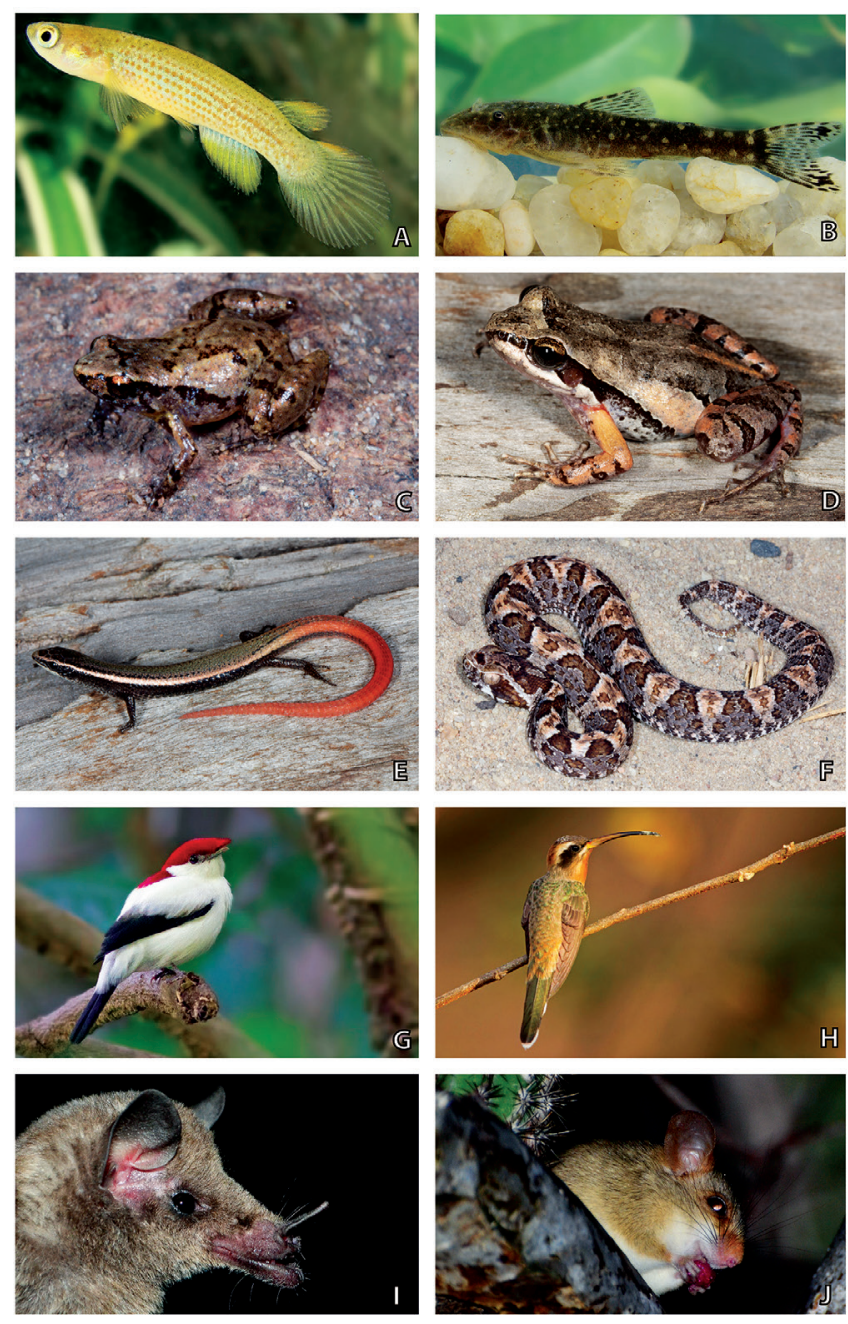

Figura 1. Algumas espécies de vertebrados endêmicos da Caatinga: A) Anablepsoides cearensis (foto Sérgio Lima), B) Parotocinclus spilurus (foto Sérgio Lima), C) Adelophryne baturitensis (foto Adrian Garda), D) Rupirana cardosoi (foto Adrian Garda), E) Psilops mucugensis (foto Adrian Garda), F) Jararaca da caatinga, Bothrops erythromelas (foto Adrian Garda), G) Soldadinho do Araripe, Antilophia bokermanni (foto Cristine Prates), H) Rabo branco de cauda larga, Anopetia gounellei (foto Ciro Albano), I) Xeronycteris vieirai, (foto Juan Caros Vargas Mena) e J) Wiedomys pyrrhorhinos (foto Juan Caros Vargas Mena)

ou controle de pragas). Após o barramento dos principais rios do semiárido, algumas espécies foram introduzidas para piscicultura, alterando a composição da biota aquática e às vezes resultando na degradação da qualidade da água.

Rosa e colaboradores [5], na primeira compilação de peixes da Caatinga, subdividiram o bioma em quatro ecorregiōes aquáticas: Maranhão-Piauí (MAPI), cuja principal bacia é a do rio Parnaíba; 
Tabela 1. Comparação do número de espécies conhecidas para o bioma Caatinga, ressaltando a mudança nos valores entre 2003 e 2017 , anos de publicação dos dois grandes compêndios sobre o bioma $[14,15]$. Número total $(\mathrm{N})$, porcentagem de endêmicos (\%E) e incremento em riqueza conhecida de 2003 para 2017 por grande grupo de vertebrados

\begin{tabular}{lccccc}
\hline & $\mathbf{2 0 0 3}$ & $\mathbf{2 0 1 7}$ & Endêmicos (N) & Endêmicos (\%E) & Aumento da riqueza (\%) \\
\hline Aves & 505 & 548 & 23 & 4,2 & 8,5 \\
Mamíferos & 143 & 183 & 11 & 6,0 & 28,0 \\
Répteis & 116 & 224 & 69 & 30,8 & 93,1 \\
Anfíbios & 56 & 98 & 20 & 20,4 & 75,0 \\
Peixes & 240 & 386 & 209 & 54,1 & 60,8 \\
\hline TOTAL & $\mathbf{1 0 6 0}$ & $\mathbf{1 4 3 9}$ & $\mathbf{3 3 2}$ & $\mathbf{2 3 , 1}$ & $\mathbf{3 5 , 8}$ \\
\hline
\end{tabular}

Nordeste Médio-Oriental (NEMO), composta por bacias menores, a maioria com regime temporário; São Francisco (SFRE), representada pela bacia homônima com longo trecho no bioma; $\mathrm{e}$ Mata Atlântica Nordeste (MANE) ou Bacias do Leste, que abrange as drenagens costeiras ao sul da foz do rio São Francisco, com trecho superior na Caatinga. Uma atualização da ictiofauna do bioma resultou em um aumento de 146 espécies $60,8 \%$ a mais que a lista anterior. Atualmente, a quantidade de espécies de peixes descritas por ecorregião é a seguinte: MAPI, 147; NEMO, 103; SFRE; 178; e MANE, 101 espécies [5]. Apesar dos esforços recentes, muitas áreas permanecem pouco exploradas, como tributários do rio São Francisco, na Bahia, e as bacias costeiras do Nordeste Médio-Oriental [5]. Do total de 371 espécies, apenas 14 ocorrem nas quatro ecorregióes, o que, juntamente com a baixa similaridade entre as mesmas (15,9\% em média), mostra como a biodiversidade de cada uma delas é única. Isso é particularmente preocupante com relação ao ambicioso projeto de transposição do rio São Francisco, que começou a operar em março 2017 (no eixo leste), bombeando água do maior rio da Caatinga para os principais rios temporários do Nordeste Médio-Oriental, nos estados do Ceará, Rio Grande do Norte, Paraíba e Pernambuco. A mudança no regime de rios (intermitentes para perenes) e a mistura de faunas de ecorregiōes bastante distintas podem resultar no empobrecimento das bacias receptoras e na homogeneização da fauna aquática.

Os rios da Caatinga também estão sujeitos aos impactos resultantes do desmatamento da vegetação ciliar que aumenta o assoreamento dos rios, às mudanças climáticas e ao uso desordenado dos recursos hídricos. Além disso, poucas unidades de conservação (UCs) da Caatinga foram criadas considerando as drenagens da paisagem e pouquíssima informação acerca das espécies de peixes em UCs da Caatinga estão disponíveis. Apesar do papel inegável das UCs na proteção da fauna de peixes do bioma, um grande número de espécies endêmicas, ameaçadas e habitat-especialistas permanecem sem proteção. Com efeito, nenhuma das 33 espécies ameaçadas de peixes da Caatinga foi registrada dentro de qualquer
UC do bioma. Assim, é necessário um maior direcionamento de políticas públicas voltadas para conservação da biota aquática e uso sustentável dos recursos hídricos, especialmente sob a perspectiva de uma maior escassez de água. No caso dos peixes é importante identificar e proteger as áreas de refúgios durante as longas estiagens, pois elas atuam como fonte para o repovoamento dos rios na estação chuvosa.

ANFÍBIOS Somente neste século tivemos as primeiras compilaçôes publicadas sobre os anfíbios da Caatinga, onde foram registradas inicialmente 48 espécies [8] e, mais recentemente, 56 [9]. A inclusão das chamadas zonas de exceção climática como parte da Caatinga, notadamente localizadas em áreas montanhosas e que abrigam relictos (espécies encontradas em certas áreas isoladas, remanescentes de fauna outrora amplamente distribuída) de Mata Atlântica e Amazônia (enclaves mésicos), praticamente dobrou esse número. Hoje reconhecemos 98 espécies de anfíbios para a Caatinga, distribuídas em 12 famílias [10]. Dentre essas espécies, 20 foram consideradas endêmicas, 14 das quais restritas aos enclaves mésicos (12 na Chapada Diamantina e quatro nos brejos de altitude do Ceará). Somente quatro espécies endêmicas estiveram associadas aos ambientes de baixada caracterizados pela típica vegetação xerofítica (vegetais que desenvolvem estruturas especiais para sobreviver em meio semiárido e desértico) da Caatinga. Outras 13 espécies tiveram distribuiçōes quase que exclusivas para o bioma Caatinga, ocorrendo apenas pontualmente fora dele, em suas margens, e poderiam ser consideradas endêmicas sob critério menos rigoroso [10].

A distribuição espacial dessas espécies de anfíbios não é homogênea na Caatinga, com valores de riqueza potencial de espécies variando de baixo a alto [10]. As áreas de menor riqueza potencial coincidiram com os resultados obtidos por Camardelli e Napoli [11]. Dentre essas áreas, teve destaque toda a margem oeste do bioma, coincidentemente fora da influência da Mata Atlântica e historicamente com menor amostragem. A maior parte das áreas quentes 
estão localizadas ao longo da margem leste do polígono que define o bioma Caatinga, próximo à zona de contato com a Mata Atlântica, entre elas as ecorregiōes do Raso da Catarina e do Planalto da Borborema, áreas de transição na Bahia e brejos de altitude nos estados de Pernambuco e Paraíba. Há ainda aquelas que são mais interioranas e presentes em zonas de altitude, como a ecorregião Chapada Diamantina, na Bahia (porção norte da Serra do Espinhaço), e a Chapada do Araripe, localizada na divisa dos estados do Ceará, Pernambuco e Piauí.

Os maiores vilóes que ameaçam os anfíbios nesse bioma são a degradação do ambiente e o desflorestamento, as áreas de proteção ambiental insuficientes, a desertificação e as mudanças climáticas. Dentre as espécies com algum grau de ameaça destacam-se aquelas situadas em áreas florestais mésicas, que também sofrem pressão da agricultura e do turismo, já que estão sob climas mais amenos e detêm apreciada beleza cênica. Além disso, como já mencionado, 14 das 20 espécies de anfíbios endêmicas para o bioma estão restritas aos enclaves mésicos e, indubitavelmente, outras tantas espécies endêmicas ainda serão descritas nos próximos anos para essas áreas (16 das 20 espécies de anfíbios endêmicos foram descritas a partir de 2006). Por outro lado, ao menos 24 espécies de anfíbios estão restritas às áreas de vegetação de caatinga localizadas em baixadas e, portanto, não contempladas pela proteção de enclaves mésicos. Considerando-se as mudanças climáticas globais, as espécies de anfíbios que ocupam áreas mais quentes e secas, intuitivamente, estarão ainda mais vulneráveis. É necessário, portanto, que sejam direcionados esforços para a conservação dessas espécies, além, é claro, daquelas presentes nos enclaves mésicos.

RÉPTEIS A fauna de répteis da Caatinga é rica em lagartos [6], serpentes [7] e anfisbenas [12]. Além disso, apesar de números aparentemente pequenos de tartarugas (7 espécies) e crocodilianos (3 espécies) [9], esses valores tornam-se significativos quando consideramos que existem 31 espécies de quelônios no Brasil e 23 de crocodilianos em todo o mundo. Atualmente são conhecidas 224 espécies de répteis para a Caatinga, 30\% delas endêmicas (Tabela 1). Dessas, temos 112 serpentes (22 endêmicas), 79 lagartos (38 endêmicos) e 23 anfisbenias ( 9 endêmicas). A riqueza conhecida praticamente dobrou desde a primeira compilação, realizada em 2003, assim como o número de espécies endêmicas reconhecidas. Esse aumento se deu tanto pela coleta de espécies de outros biomas nas margens ou em ilhas úmidas dentro da Caatinga, como pela descrição de novas espécies exclusivas do bioma, principalmente de lagartos. Com efeito, lagartos possuem níveis de endemismo similares aos de peixes, na casa do $50 \%$ (Tabela 1), e a riqueza potencial estimada de lagartos para o bioma indica as Paleodunas do Rio São Fran- cisco e regiōes de Pernambuco, Paraíba e Rio Grande do Norte como as potencialmente mais ricas em espécies na Caatinga.

Ao contrário do que vemos para anfíbios, a maioria das espécies de lagartos endêmicas não está restrita a enclaves mésicos, sendo concentradas principalmente nas paleodunas do rio São Francisco e na região do raso da Catarina [6]. As espécies de lagartos podem ser agrupadas de acordo com seus padrões de distribuição: i) ampla distribuição na Caatinga (16 espécies); ii) distribuição relictual, para espécies que ocupam hoje áreas que são remanescentes de destruições maiores no passado (15 espécies); iii) distribuições limitadas dentro da Caatinga (6 espécies); iv) espécies relacionadas às paleodunas do rio São Francisco (12 espécies); v) espécies limitadas a afloramentos rochosos da Serra do Espinhaço (8 espécies); e vi) espécies de biomas adjacentes, como cerrados e restingas (9 espécies).

Para serpentes, outro grupo bastante diverso na Caatinga, um efeito da topografia da região parece evidente, com grupos de espécies associados a áreas altas e outros a regióes de baixada [7]. Embora esteja clara a importância dos ambientes adjacentes e úmidos para a diversidade de répteis da Caatinga, está cada vez mais evidente que, antes considerada depauperada e pobre em espécies endêmicas, ela na verdade abriga riqueza e endemismo comparáveis a outros biomas neotropicais.

Trabalhos pioneiros dividiram a Caatinga em oito ecorregiões com base em informações sobre solo, clima, vegetação, geomorfologia, geologia e conhecimento de especialistas [13]. Ecorregiōes são unidades grandes de terra e água definidas por fatores bióticos e abióticos que influenciam as comunidades naturais que lá ocorrem. As ecorregiōes servem como unidades de planejamento para a criação de unidades de conservação, por exemplo. Para lagartos e serpentes, grande parte das ecorregiōes da Caatinga definidas segundo os critérios acima são recuperadas quando comparamos os níveis de espécies endêmicas dentro do bioma $[6,7,11]$. Ou seja, a distribuição de espécies endêmicas de répteis suporta as ecorregiōes e centros de endemismo sugeridos para outros grupos de seres vivos e com metodologias distintas. Essa congruência é fundamental para o planejamento sistemático da conservação da Caatinga, pois permite subdividir o bioma em áreas relativamente independentes e, assim, garantir a proteção.

AVES Hoje conhecemos 548 espécies de aves para a Caatinga, um número que vem crescendo principalmente devido à colaboração de pessoas que praticam a observação desses animais, o que demonstra a importância da ciência-cidadã no fornecimento de informações sobre a biodiversidade em regiōes tropicais. Um total de 509 espécies são consideradas residentes, porque se reproduzem na região; 22 espécies são migrantes visitantes do hemisfério norte e 10 são migrantes de regiōes mais ao sul da América do Sul. Ainda, algumas das espécies que se reproduzem na região realizam migrações ou 
movimentos regionais, de acordo com a distribuição das chuvas [3]. A reprodução da grande maioria das espécies de aves na Caatinga ocorre durante o período chuvoso. Embora a precipitação também se relacione com uma maior abundância de recursos, como alimentos, o que mais influencia diretamente a frequência reprodutiva das aves é o aumento da quantidade de vegetação nesse período [3].

Do total, 67 espécies ou subspécies se originaram na região e são categorizadas em três grandes grupos: 1) um composto por táxons com distribuição restrita a enclaves de habitats dentro do limite da Caatinga (ex. soldadinho-do-araripe - Antilophia bokermanni); 2) outro composto por táxons que ocorrem nos ambientes deciduais, mas com distribuição relativamente ampla na região (ex. rabo-branco-de-cauda-larga - Anopetia gounellei); 3) e, por fim, o grupo de espécies cuja ocorrência é associada aos ambientes deciduais encontrados na Caatinga, mas que podem ser também registrados em manchas de florestas secas adjacentes à região (ex. arapaçu-do-nordeste - Xiphocolaptes falcirostris) [3]. Ainda, existem outras espécies consideradas originalmente endêmicas, mas que expandiram rapidamente sua distribuição devido à substituição de florestas ou savanas em regiōes adjacentes, ou mesmo por causa de solturas devido ao tráfico ilegal no Brasil (ex. corrupião - Icterus jamacaii). De acordo com informações moleculares, as origens das espécies de aves endêmicas da Caatinga datam da metade do Mioceno até o Holoceno. Alguns desses endemismos são associados à vegetação mais arbustiva e aberta, mas outros ocorrem estritamente em ambientes florestais, sejam deciduais ou enclaves de florestas úmidas [3]. Isso sugere que a existência de um mosaico de fisionomias com vegetação mais aberta e vegetação florestal não é recente na Caatinga.

As maiores riquezas de espécies, entre 210 e 259, foram registradas em localidades de transição e com a ocorrência de uma heterogeneidade de habitats. Ainda, é extremamente importante entender que essas localidades correspondem a ambientes em um bom estado de conservação quando comparadas à maior parte da região Nordeste, como por exemplo no sudeste do Piauí. Vastas áreas dominadas por vegetação arbustiva podem ser observadas em uma grande região da Caatinga, principalmente associadas às depressões entre serras e planaltos. Nessas localidades, a riqueza registrada variou entre 121 e 145 espécies de aves. As menores riquezas, entre 90 e 120 espécies, foram registradas em localidades em que a paisagem mantinha vegetação arbustiva, mas com grandes áreas abertas provenientes da modificação antrópica para pastagens ou campos agrícolas [3].

Em uma região dominada por terras degradadas e com um clima mais quente e seco, espera-se que poucas espécies de aves consigam manter populações viáveis com as modificações oriundas da ação do ser humano. Para estimar como as espécies podem lidar com transformações do uso da terra e mudanças climáticas, pesquisadores usaram a experiência de campo e pesquisa bibliográfica para classificar cada espécie em três categorias de capacidade adaptativa: (a) alta capacidade, espécies que podem ser encontradas em ecossistemas antropogênicos distantes de qualquer ecossistema natural; (b) capacidade média, espécies que podem ser encontradas em ecossistemas antropogênicos se e somente se esses ecossistemas estiverem próximos a ecossistemas naturais; e (c) baixa capacidade, espécies encontradas apenas em paisagens compostas por ecossistemas intactos ou quase intocados. Com isso, foi verificado que 162 espécies tinham alta capacidade adaptativa, 207 tinham capacidade adaptativa média e 179 apresentavam baixa capacidade adaptativa na Caatinga [3]. Das 35 espécies classificadas como ameaçadas pela IUCN (União Internacional para a Conservação da Natureza) ou pelo governo brasileiro, a maioria delas $(83 \%)$ possui baixa capacidade adaptativa, com $17 \%$ exibindo capacidade adaptativa média. A conservação a longo prazo dessa avifauna regional depende da proteção da heterogeneidade ambiental em toda a região. A proteção e restauração dos ecossistemas naturais nos topos e encostas dos planaltos isolados, assim como nos vales dos rios, é a espinha dorsal de um plano de conservação da avifauna regional [3].

MAMÍFEROS Os mamíferos da Caatinga são os menos estudados de todas as grandes regiōes ecológicas do Brasil [4]. Ainda assim, a fauna conhecida para a Caatinga hoje é de 183 espécies, 11 das quais são endêmicas [4], números que correspondem a um incremento significativo na riqueza conhecida nos últimos 14 anos (Tabela 1). Grande parte da diversidade é representada por roedores e morcegos e existem animais com distribuições geográficas variadas, de espécies restritas a espécies de ampla distribuição. Os primeiros trabalhos, de antes da década de 1990, consideraram a Caatinga pobre em espécies, com baixo endemismo, onde a maioria de suas espécies era um subconjunto da fauna do Cerrado e que as espécies da Caatinga não tinham adaptaçôes fisiológicas ao clima semiárido (apenas mudanças comportamentais para tolerar ambientes hostis).

Hoje sabemos que ainda que a Caatinga possua riqueza e endemismo menores que os biomas vizinhos, está longe de ser considerada pobre e sem identidade. Além disso, os valores de riqueza e endemismo vem aumentado ano a ano com a descrição de novas espécies, revisões taxonômicas e expansão de distribuição de espécies de ambientes vizinhos. Ainda, o padrão de compartilhamento de espécies é mais complexo do que antes sugerido, com espécies sendo também compartilhadas com a Amazônia, a Floresta Atlântica e o Chaco. Igualmente, as espécies não estão distribuídas de forma homogênea na Caatinga, existem padrões distintos de riqueza e uma história biogeográfica que apenas agora começa a ser descoberta. As espécies endêmicas, por sua vez, possuem associações com diferentes habitats e origens evolutivas diferentes, o que implica em uma história necessariamente complexa da origem e da evolução da Caatinga como um todo. A hipótese da ausência de adaptaçôes fisiológicas, por sua vez, é em geral confirmada. Isso porque apesar de existirem algumas, elas não são, nem de longe, tão extremas como as de roedores de desertos, por exemplo [4].

As ordens mais representativas do bioma são Chiroptera (morcegos, com 90 espécies) e Rodentia (roedores, com 41 espécies), 
totalizando $71 \%$ da riqueza de mamíferos da Caatinga e 10 das 11 espécies endêmicas [4]. Para alguns grupos, grande parte das espécies brasileiras ocorre na Caatinga, como Carnivora (48\% das espécies), Chiroptera (50\%) e Cingulata (tatus, 64\%). Apesar de menos de $20 \%$ dos roedores brasileiros ocorrerem na Caatinga, o grupo é particularmente interessante, pois 7 das 11 espécies endêmicas do bioma são Rodentia. Em contraste, a fauna de morcegos da Caatinga é mais rica e bastante representativa da fauna de Chiroptera do Brasil, mas curiosamente possui apenas três espécies endêmicas [4].

Dados básicos sobre a ecologia de mamíferos na Caatinga são muito raros e, em geral, o pouco que sabemos está restrito a pequenos mamíferos, com dados para mamíferos de médio e grande porte e morcegos ainda mais escassos [4]. Com o pouco que existe na literatura (e como seria esperado), sabemos que os mamíferos desse bioma dependem e respondem diretamente ao regime de chuvas. Em anos com chuvas mais intensas e, portanto, com maior disponibilidade de recursos alimentares, as populações de roedores costumam aumentar significativamente, dando origem a fenômenos conhecidos como ratadas, marcados por aumentos explosivos na abundância de roedores em um curto espaço de tempo [4]. Fisiologicamente, os dados atuais sugerem que existem poucas adaptações de mamíferos da Caatinga ao estresse hídrico e que mudanças comportamentais parecem ser chave para esses animais lidarem com as limitaçôes na disponibilidade de água no bioma. Com efeito, algumas espécies mudam para ambientes mais úmidos dentro da Caatinga em períodos de escassez, enquanto outras alteram seu regime diário de atividade e dieta. Claramente, ainda são necessários muitos estudos para compreender a ecologia e o comportamento dos mamíferos da Caatinga.

CONCLUSÕES Um número expressivo de espécies de vertebrados ocorre na Caatinga. O aumento vertiginoso no número de espécies conhecidas para o bioma nos últimos 15 anos mostra o quão pouco conhecíamos da Caatinga. Além disso, as taxas de descrição de novas espécies e extensão de distribuições geográficas indicam que ainda há muito por fazer e que, portanto, esses números são subestimados. Somado a isso, uma rede de unidades de conservação pequena, a pressão humana resultante de índices de desenvolvimento humano baixos e as iminentes mudanças climáticas, tornam a conservação dos vertebrados na Caatinga desafiadora.

Adrian Antonio Garda éprofessor associado do Departamento de Botânica e Zoologia da
Universidade Federal do Rio Grande do Norte (UFRN), Natal-RN.

Marília Bruzzi Lion é pesquisadora associada e bolsista PNPD do Programa de PósGraduação em Ecologia da UFRN, Natal-RN.

Sérgio Maia de Queiroz Lima é professor adjunto do Departamento de Botânica e Zoologia da UFRN, Natal-RN.

Daniel Oliveira Mesquita é professor associado do Departamento de Sistemática e Ecologia da Universidade Federal da Paraíba (UFPB), João Pessoa-PB.

Helder Farias Pereira de Araujo é professor adjunto do Departamento de Ciências Biológicas da UFPB, Areia-PB.

Marcelo Felgueiras Napoli é professor associado do Departamento de Zoologia da Universidade Federal da Bahia (UFBA), Salvador-BA.

\section{REFERÊNCIAS}

1. Fonseca, C. R.; Antongiovanni, M.; Matsumoto, M.; Bernard, E.; Venticinque, E. M. In: Biodiversity, ecosystems services and sustainable development in Caatinga: the largest tropical dry forest region in South America. Filho, E. M.; Leal, I. R.; Tabarelli, M. (eds.) Springer-Verlag, Berlin, 2017, pp. 429-443.

2. Gehara, M. et al. "Estimating synchronous demographic changes across populations using $\mathrm{hABC}$ and its application for a herpetological community from northeastern Brazil". Molecular Ecology 26, 4756-4771,2017.

3. Araujo, H. F. P.; Silva, J. M. C. In: Biodiversity, ecosystems services and sustainable development in Caatinga: the largest tropical dry forest region in South America. Filho, E. M.; Leal, I. R.; Tabarelli, M. (eds.) Springer-Verlag, Berlin, 2017, pp. 210.

4. Carmignotto, A. P.; Astúa, D. In: Biodiversity, ecosystems services and sustainable development in Caatinga: the largest tropical dry forest region in South America. Filho, E. M.; Leal, I. R.; Tabarelli, M. (eds.) Springer-Verlag, Berlin, 2017, pp. 211-254.

5. Lima, S. M. Q.; Ramos, T.P. A.; Da Silva, M. J.; Rosa, R. S. In:Biodiversity, ecosystems services and sustainable development in Caatinga: the largest tropical dry forest region in South America. Filho, E. M.; Leal, I. R.; Tabarelli, M. (eds.) Springer-Verlag, Berlin, 2017, pp. 97-131.

6. Mesquita, D. O.; Costa, G. C.; Garda, A. A.; Delfim, F. R. In: Biodiversity, ecosystems services and sustainable development in Caatinga: the largest tropical dry forest region in South America. Filho, E. M.; Leal, I. R.; Tabarelli, M. (eds.) Springer-Verlag, Berlin, 2017.

7. Guedes, T. B.; Sawaya, R. J.; Nogueira, C. d. C. “Biogeography, vicariance and conservation of snakes of the neglected and endangered Caatinga region, north-eastern Brazil". Journal of Biogeography 41, 919-931,2014.

8. Rodrigues, M. T. In: Ecologia e conservação da Caatinga. Leal, I. R.; Tabarelli, M.; Silva, J. M. C. (eds.) Editora Universitária da UFPE, Recife, 2003, pp. $181 \oplus 236$.

9. Albuquerque, U. P. et al., "Caatinga revisited: ecology and conservation of an important seasonal dry forest". The Scientific World Journal 2012, 205182,2012.

10. Garda, A. A. et al. In: Biodiversity, ecosystems services and sustainable development in Caatinga: the largest tropical dry forest region in South America. Filho, E. M.; Leal, I. R.; Tabarelli, M. (eds.) SpringerVerlag, Berlin, 2017, pp. 133-149.

11. Camardelli, M.; Napoli, M. F. "Amphibian conservation in the Caatinga biome and semiarid region of Brazil". Herpetologica 68, 31-47, 2012.

12. Ribeiro, L.B.; Gomides, S.C.; Costa,H.C. "Anew species of Amphisbaena from Northeastern Brazil (Squamata: Amphisbaenidae)". Journal of Herpetology 52, 234-241, 2018.

13. Velloso, A. L.; Sampaio, E. V. S. B.; Pareyn, S. G. S. Ecorregiões propostas para o bioma Caatinga. Instituto de Conservação Ambiental The Nature Conservancy do Brasil, 2002, pp. 76.

14. Leal, I. R.; Tabarelli, M.; Silva, J. M. C. Ecologia e conservação da Caatinga. Universidade Federal de Pernambuco, Recife, 2003, pp. 806.

15. Silva, J. M. C. d.; Leal, I. R.; Tabarelli, M. Biodiversity, ecosystems services and sustainable development in Caatinga: the largest tropical dry forest region in South America. Springer-Verlag, Berim, 2017, pp. 482. 